\title{
Melatonin modulates hypoxia-induced changes of rat brain excitability
}

\author{
Dana Maresova ${ }^{1}$, Vladimir Riljak ${ }^{1}$ and Jan Mares $^{2}$ \\ ${ }^{1}$ Institute of Physiology, ${ }^{\text {st }}$ Faculty of Medicine, Charles University in Prague, Czech Republic; E-mail: vladimir.riljak@lf1.cuni.cz \\ ${ }^{2}$ Department of Normal, Pathological and Clinical Physiology, $3^{\text {rd }}$ Medical School, Charles University in Prague, Czech Republic
}

\begin{abstract}
The aim of our study was to test the hypothesis, if melatonin pre-treatment (in dose of $100 \mathrm{mg} / \mathrm{kg}$ ) can influence the changes of brain function after short-term hypoxia exposition (simulated altitude $9000 \mathrm{~m}$ ) in young immature rats. Experiments were performed on freely moving 12-, 25- and 35-day-old male Wistar rats. One hour prior to hypoxia exposition, animals were pre-treated with melatonin and 24 hours after hypoxia cortical afterdischarges (ADs) were elicited by repeated stimulation of the right sensorimotor cortex. The duration of evoked ADs and shape of evoked graphoelements was monitored. Short-term exposure to hypoxic conditions resulted in significantly shorter ADs duration in 12-day-old rats after stimulations (except the $2^{\text {nd }}$ one stimulation) compared to control group. Administration of melatonin prolonged the duration of ADs after all stimulations except the $1^{\text {st }}$ one. Analysis of the duration ADs revealed no significant changes, either after the exposition to hypobaric hypoxia or after melatonin administration in 25- and 35-day-old animals. Effects and mechanisms of melatonin action on the brain seizure susceptibility and the possible beneficial role of that treatment in hypoxic brain damage are discussed.
\end{abstract}

Key words: Melatonin — Short-term hypoxia — Free radicals — Epileptogenesis

\section{Introduction}

Hypoxia and its effects in central nervous system have been studied extensively for many years because its clinical importance in many medical issues (Vannuci and Vannuci 2005). It has been postulated, that significant "hypoxia-response" differences between adult and immature organism exist and that the final effect of hypoxic event depends not only on the intensity and duration of the period of oxygen deficiency, but also on the ontogenetic stage of subject exposed to hypoxia (Golan and Huleihel 2006). Extent of oxygen deficiency (brain ischemia) is classified as the focal and global one. The former mentioned term means the interruption of the blood supply to some portion of the brain (hemorrhagic or ischemic stroke), the latter term describes interruption of oxygen delivery into the whole brain (cardiac arrest, decreased partial pressure of oxygen in surrounding atmosphere) and significantly affect the whole body homeostasis, with many consequences (activation of sympathoadrenal

Correspondence to: Vladimir Riljak, Institute of Physiology, $1^{\text {st }}$ Faculty of Medicine, Charles University in Prague, Albertov 5, 12800 Prague 2, Czech Republic

E-mail: vladimir.riljak@lf1.cuni.cz system etc.) (Ginsberg and Busto 1989; Lipton 1999; Hartman et al. 2005; Cervantes et al. 2008). In the following text the term hypoxia we use for global decrease of oxygen supply to the brain, being aware that it is certain simplification.

In the central nervous system hypoxia affects the neuronal homeostasis and finally it can lead to excitotoxic damage and cell death. Excitotoxic pathways are triggered by excessive glutamate release. It causes neuronal depolarization (so called glutamate loop) and by increased intracellular calcium it brings about a functional overload of neuronal circuits which can eventually result in cell death (Doble 1999). Another mechanism of a severe hypoxic brain damage is the "reperfusion or reoxygenation phase". During that period several factors, mainly the reactive oxygen and reactive nitrogen species cause a molecular tissue impairment and neuronal and glial cell loss (Fellman and Raivio 1997; Buonocore et al. 2001; Carloni et al. 2008). It is believed that brain tissue is relatively deficient in antioxidative defence system. It is related to the high consumption of oxygen in the brain tissue which is significantly greater than in other tissues (brain utilizes $20 \%$ of inhaled oxygen but it forms only $2 \%$ of the total body weight). At resting conditions brain generates large quantity of injurious free oxygen and nitrogen species and extreme conditions like hypoxia and 
recovery from hypoxia can easily disrupt the free radicals homeostasis. It can cause learning impairment, memory deficits etc. (Fellman and Raivio 1997; Buonocore et al. 2001; Kaur and Ling 2008).

Melatonin (N-acetyl-5-methoxytryptamine) is a hormone of pineal gland with almost exclusively night production and mainly unique light/night production suggestibility. Discovering the possible antioxidant features of melatonin leads to arise of series of studies, differently designed to test the melatonin-related antioxidant capacity. Melatonin and its metabolites are capable to neutralize oxygen-based toxic agents like hydroxyl radical, hydrogen peroxide, hypochlorous acid or singlet oxygen; additionally melatonin stimulates a number of antioxidative enzymes (glutathione peroxidase, glutathione reductase) (Reiter et al. 2003, 2005; Tan et al. 2005; Matějovská et al. 2008). With respect to the latter we hypothesized, that melatonin could modify the brain susceptibility and excitability of tested young animals, by scavenging the free radicals, produced in excess by acute hypoxia stimulus and during the phase of recovery ("reperfusion"). Melatonin seems to be very useful drug, because it is able quickly enter the central nervous system via penetrating the blood brain barrier and a significant concentration increase could be detected in CNS in few minutes after the intraperitoneal administration.

According to references in literature (Buonocore at al. 2001; Carloni et al. 2008; Cervantes et al. 2008) we tested the hypothesis that pre-treatment with a single very high dose of melatonin $(100 \mathrm{mg} / \mathrm{kg})$ could be beneficial for the preservation of nerve tissue during the hypoxic conditions and thus might to modify the development of the pathogenetic processes, mentioned above.

\section{Materials and Methods}

\section{Animals}

All experiments were carried out in accordance with the European Communities Council Directive of 24 November 1986 (86/609/EEC) and in agreement with the guidelines of the Animal Protection Law of the Czech Republic.

Experiments were performed on freely moving 12-, 25and 35-day-old Wistar rats of our own breed. They were housed at a constant temperature $\left(23 \pm 1^{\circ} \mathrm{C}\right)$ and relative humidity $(60 \%)$ with a fixed $12 \mathrm{~h}$ light/dark cycle and fed with food and water ad libitum. There were at least 8 animals in each experimental group used for statistical analysis.

\section{Experimental substances and procedures}

All drugs were applied intraperitonealy $\left(11^{\text {th }}, 24^{\text {th }}\right.$ or $34^{\text {th }}$ day). Melatonin was dissolved in ethanol and then injected in the dose $100 \mathrm{mg}$ per kilogram of animal body weight, recalculated volume: $0.02 \mathrm{ml}$ of the solution per $10 \mathrm{~g}$ of animal body weight. Ethanol was applied in equal volumes.

A 60 min rest after the injection of any substance was followed by exposure to $6.4 \mathrm{kPa}$ hypobaric hypoxia, i.e. to the simulated altitude of $9000 \mathrm{~m}$, which was reached in $2 \mathrm{~min}$ $(30 \mathrm{kPa} / \mathrm{min})$ and lasted $60 \mathrm{~min}$. After returning to ambient air pressure (approximately $101 \mathrm{kPa}$ ) with the speed of 30 $\mathrm{kPa} / \mathrm{min}$, animals were left to recover at resting conditions for 24 hours. After this period (this recovery time of 24 hours was kept for the entire experimental group after any drug administration) six silver electrodes were implanted through the cranium under deep anaesthesia: two stimulation electrodes (right sensorimotor cortex), three registration electrodes (left sensorimotor cortex, left and right visual cortex) and reference electrode (prefrontal cortex). Recording and other experimental manipulations were carried out after the recovery of righting reflexes (i.e. approximately 15 min after the surgery).

At the age of $12^{\text {th }}, 25^{\text {th }}$ or $35^{\text {th }}$ day the cortical afterdischarges (ADs) were elicited by stimulation of the right sensorimotor cortex. We used constant current stimulation (bipolar pulses - pulse period $1 \mathrm{~ms}$; duration of stimulation $15 \mathrm{~s}$; frequency $8 \mathrm{~Hz}$; intensity $3-5 \mathrm{~mA}$, which is sufficient for ADs eliciting). The basic stimulation intensity level was set at $3 \mathrm{~mA}$. In case of no response, another stimulation of $4 \mathrm{~mA}$ was used $5 \mathrm{~min}$ after the first stimulation. The process was similarly repeated with $5 \mathrm{~mA}$ stimulation. Finally, if no epileptic graphoelements appeared after the $5 \mathrm{~mA}$ stimulation, the animal was excluded from the experiment. If a distinct response (epileptic graphoelements) was recorded, the stimulation was repeated five times at one-minute intervals (timed from the end of each seizure to the beginning of the next stimulation). The duration of evoked ADs and shape of evoked graphoelements was monitored (for monitoring the EEG EPAS/COMPEG24 has been used). The paired, unpaired $t$-test and ANOVA in GraphPadPrism were used for evaluation of the results.

Animals (in all age groups) were randomly assigned into the following subgroups: Control group (C) - received single injection of ethanol solution and was not exposed to hypoxia; Control/Hypoxia group (C/HYP) - received a single injection of ethanol $1 \mathrm{~h}$ prior to hypoxia exposition; Melatonin group (M/HYP) - received a single injection of melatonin $1 \mathrm{~h}$ prior to hypoxia exposition.

\section{Results}

\section{2-day-old animals}

Analysis of electrocorticogram (ECoG) revealed that typical type of observed ADs was a train of fast sharp waves, while 


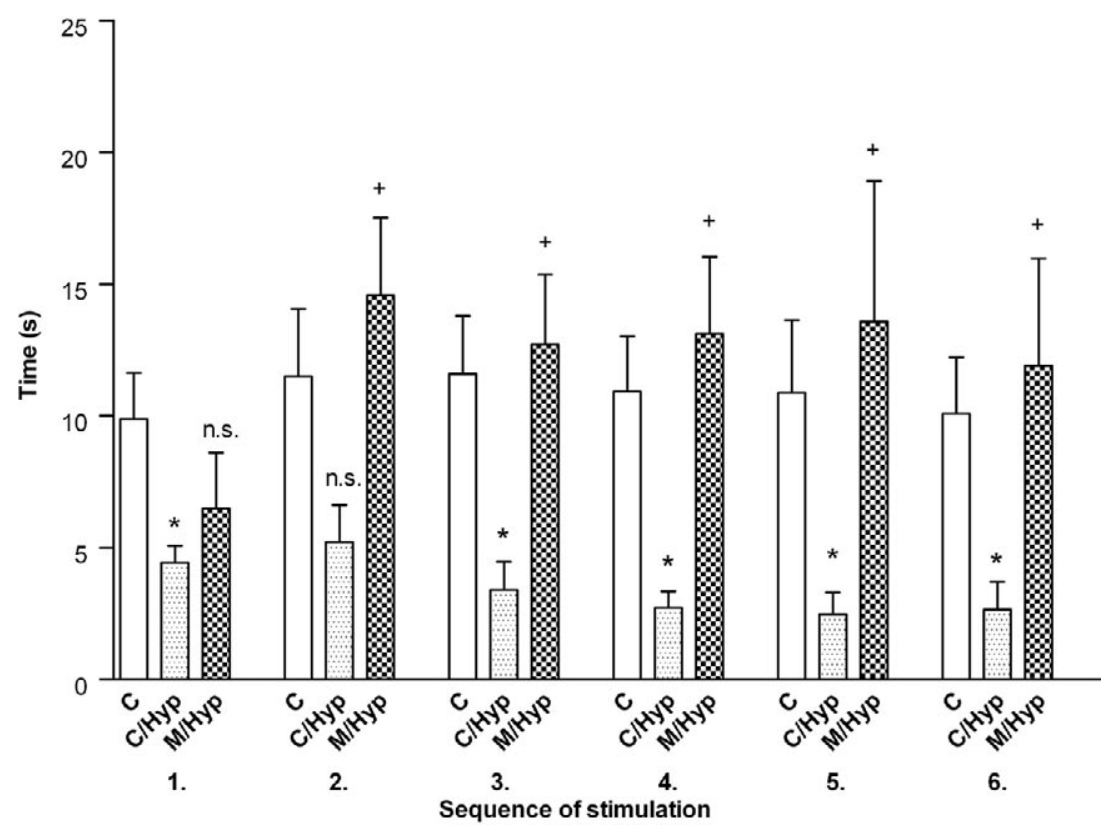

Figure 1. Duration of ADs in 12-day-old rats. 1-6, sequence of stimulation; white columns - rats treated with dissolvent (C); spotted columns - rats exposed to hypoxia and pre-treated with dissolvent (C/Hyp); hatched columns - rats exposed to hypoxia and pre-treated by melatonin $(\mathrm{M} / \mathrm{Hyp}) ;{ }^{*}$ results significant at $p<0.05$ obtained by comparison $\mathrm{C}$ and $\mathrm{C} / \mathrm{Hyp} ;{ }^{+}$results significant at $p<0.05$ obtained by comparison C/Hyp and M/Hyp.

spikes of low amplitude were rarely recorded. The duration of ADs after the $1^{\text {st }}$ stimulation was $9.87 \pm 1.78 \mathrm{~s}$. The duration of ADs after $2^{\text {nd }}, 3^{\text {rd }}, 4^{\text {th }}, 5^{\text {th }}$ and $6^{\text {th }}$ stimulation did not revealed any significant change, compared to the ADs duration after $1^{\text {st }}$ stimulation. The results show the fact, that repeating the stimulation doesn't lead to any change in cortical excitability and the response of immature cortex remain unchanged even the electrical pulses are repeated.

Short-term exposure to hypoxic conditions brought about significant shortening of evoked ADs durations (except the $2^{\text {nd }}$ one) compared to those in control group $(p<0.05)$ (the recording was performed $24 \mathrm{~h}$ after the exposition to hypoxia). Administration of melatonin prolonged the duration of ADs after the repeated stimulation except the $1^{\text {st }}$ one (Fig. 1).

\section{5-day-old animals}

In 25-day-old animals ECoG graphoelements consisting of spike-and-waves prevailed. A small amount of biphasic bursts superposed on slow waves also appeared. The analysis of ADs duration did not detect any significant changes either after exposition of hypobaric hypoxia or after hypoxia with melatonin pre-treatment. Repeating the electrical stimulation leads to the shortening of ADs, it means the $2^{\text {nd }}, 3^{\text {rd }}$ and $4^{\text {th }}$ stimulation produce significantly shorter ADs, while $5^{\text {th }}$ and $6^{\text {th }}$ stimulation produce ADs, which are of same statisti- cal duration like the ADs after the first electrical impulse. This effect was observed as well in hypoxia group as in group which was pre-treated by melatonin.

\section{5-day-old animals}

The types of ECoG graphoelements were similar to those detected in 25-day-old group with the spike and waves domination. The analysis of ADs duration did not detect any significant changes either after exposition of hypobaric hypoxia or after melatonin administration. ADs duration statistically did not detect any significant changes either after exposition of hypobaric hypoxia or after hypoxia with melatonin pre-treatment. Repeating the electrical stimulation leads to the shortening of ADs, it means the $2^{\text {nd }}, 3^{\text {th }}$ and $4^{\text {th }}$ stimulation produce significantly shorter ADs, while $5^{\text {th }}$ and $6^{\text {th }}$ stimulation produce ADs of same statistical duration like the ADs after the first electrical impulse (Fig. 2). This effect was observed in hypoxia group as well as in group which was pre-treated by melatonin.

\section{Discussion}

The aim of our study was to test the hypothesis, if melatonin pre-treatment can influence changes of brain function after short-term exposition to hypoxia in young immature rats. 


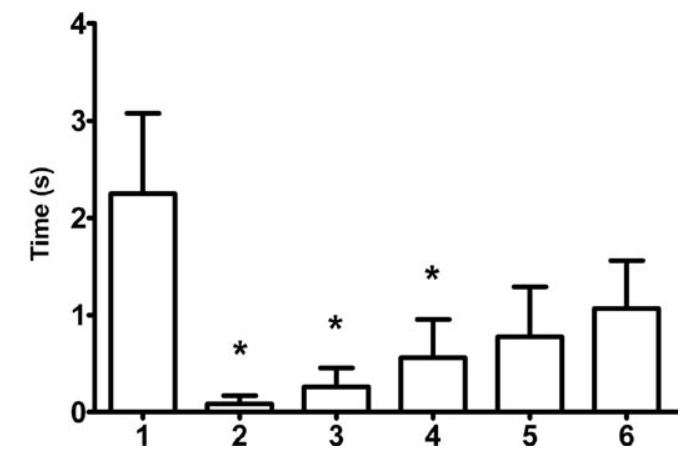

Figure 2. Duration of ADs in 35-day-old rats treated only with dissolvent. The effect of postictal inhibition ("U-shape") is shown. $1-6$, sequence of stimulation; ${ }^{\star}$ results significant at $p<0.05$.

To test the susceptibility of central nervous system and possible effect of melatonin we decided to use the method of evoked cortical ADs, because it effectively tests and senses the susceptibility and excitability of brain tissue and can register changes of the brain homeostasis (Reiter et al. 2003; Kalinčík and Marešová 2005; Vannuci and Vannuci 2005; Golan and Huleihel 2006; Cervantes et al. 2008). This susceptibility to seizures is maintained (and under pathological conditions kept) by equilibrium/disequilibrium of excitatory and inhibitory mechanisms. It is well described that the inhibitory mechanisms are developing during the period of early ontogenesis and this could explain the fact, that 12-day-old animal are unable to prevent prolongation of ADs after repeated stimulation (Langmeier and Marešová 2005). Fig. 3 demonstrates comparison of the $1^{\text {st }}$ ADs duration in entire age group and acknowledges that $\mathrm{ADs}$ is significantly related to development of excitatory/inhibitory neuronal systems. The inhibitory systems are probably well developed in older animals which are able to prevent prolongation of the next

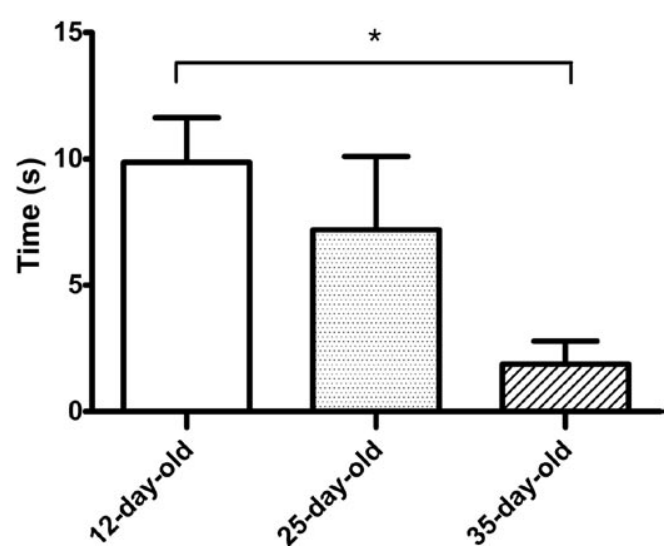

Figure 3. Duration of the $1^{\text {st }} \mathrm{AD}$ in 12-, 25- and 35-day-old animals treated with dissolvent only. ${ }^{*}$ results significant at $p<0.05$.
ADs after repeated stimulation. The typical excitability and ADs duration when compared to those one observed after the first stimulation is restored after the $4^{\text {th }}$ stimulation. The graphical representation of such relation has so called "Ushape" (Fig. 2). This physiological regulatory effect remained unaffected by melatonin pre-treatment. It has to be note, that the excitability was tested $24 \mathrm{~h}$ after the hypoxic insult and the older experimental animals are probably able to restore injured brain homeostasis, whereas the neuronal tissue of the youngest animals is heavily damaged. The short-term hypobaric hypoxia decreased the duration of $\mathrm{ADs}$ in 12day-old animals. One of possible mechanism is the depletion of cellular energetic reserves (Vannuci and Vannuci 2005). Hypoxic conditions alter the ionic equilibrium at the neuronal membrane and cells become more susceptible to depolarization (Doble 1999; Vannuci and Vannuci 2005). The excessive and repeating depolarization could lead to the cell death by excitotoxic mechanisms (Doble 1999). The immature brain is probably very sensitive to any disturbance of brain microenvironment and the ADs shortening can be explained by neuronal extinction, which affects cells that are unable to respond to stimulation by seizures. The melatonin administration, leading to ADs prolongation (compared to group of animal exposed to hypoxia condition and without melatonin pre-treatment) could represent a positive effect of melatonin, which restores the original brain tissue homeostasis, it means that melatonin influence the electrical excitability of cortex, when administered before hypoxia exposition, and the response of cortex is than very similar to response produced by animals which were unaffected by hypoxia condition.

Melatonin has been repeatedly described as a powerful scavenger for reactive oxygen species (ROS) (Reiter et al. 2003; Reiter et al. 2005) and it is well documented that hypoxia and mainly the phase of reperfusion is combined with excessive ROS generation (Cervantes 2008). In older animal groups ADs duration remains unaffected both after hypoxia or melatonin treatment, while the youngest animals seemed to profit from prophylactic administration of melatonin. The immature brain is highly vulnerable to oxidative stress and the antioxidant possibilities are very poor; it is very likely that the antioxidant actions of melatonin could provide the neuroprotection. This strong age-dependency should focus the attention on the melatonin use in very early ontogenesis and it is very important finding of this experiment. Melatonin (because of its no toxic side-effects) could prevent or improve the treatment of perinatal hypoxia and its consequences. Very important was the observation, that melatonin does not affect the postictal inhibition in the more mature animals. This underlies the hypothesis, that melatonin cannot affect brain tissue via pro-excitatory mechanisms and by worsening the brain micro-environment. In relation to that we can speculate and prepare next experiments whether the prenatal 
administration of melatonin to pregnant rats could reveal the melatonin protective effect on the perinatal hypoxic injury of the brain.

Acknowledgement. This work was supported by grants: MSM 00216208 16, GACR 305/09/P136 and GACR 309/09/0406.

\section{References}

Buonocore G., Perrone S., Bracci R. (2001): Free radicals and brain damage in the newborn. Biol. Neonate 79, 180-186; doi:10.1159/000047088

Carloni S., Perrone S., Buonocore G., Longini M., Proietti F., Balduini W. (2008): Melatonin protects from the long-term consequences of a neonatal hypoxic-ischemic brain injury in rats. J. Pineal Res. 44, 157-164; doi:10.1111/j.1600079X.2007.00503.X

Cervantes M., Morali G., Letepichia-Vallejo G. (2008): Melatonin and ischemia reperfusion injury of the brain. J. Pineal Res. 45, 1-7; doi:10.1111/j.1600-079X.2007.00551.x

Doble A. (1999): The role of excitotoxicity in neurodegenerative disease: Implications in therapy. Pharmacol. Ther. 81, 163-221; doi:10.1016/S0163-7258(98)00042-4

Fellman V., Raivio K. O. (1997): Reperfusion injury as the mechanism of brain damage after perinatal asphyxia. Pediatr. Res. 41, 599-607; doi:10.1203/00006450-199705000-00001

Ginsberg M. D., Busto R. (1989): Rodent models of cerebral ischemia. Stroke 20, 1627-1642

Golan H., Huleihel M. (2006): The effect of prenatal hypoxia on brain development: short- and long-term consequences demonstrated in rodent models. Dev. Sci. 9, 338-349; doi:10.1111/j.1467-7687.2006.00498.x

Hartman R. E., Lee J. M., Zipfel G. J., Wozniak D. F. (2005): Characterizing learning deficits and hippocampal neuron loss following transient global cerebral ischemia in rats. Brain Res. 1043, 48-56; doi:10.1016/ j.brainres.2005.02.030

Kalinčík T., Marešová D. (2005): Influence of magnesium sulphate on evoked activity of rat brain after exposure to shortterm hypoxia. Physiol. Res. 54, 229-234

Kaur C., Ling E. A. (2008): Antioxidants and neuroprotection in the adult and developing central nervous system. Curr. Med. Chem. 15, 3068-3080; doi:10.2174/ 092986708786848640

Langmeier M, Maresová D. (2005): Intermittent hypobaric hypoxia during development--morphological and functional changes in the neocortex. Prague Med. Rep. 106, 275-82

Lipton P. (1999): Ischemic cell death in brain neurons. Physiol. Rev. 79, 1431-1568

Matějovská I., Bernášková K., Krýsl D., Mareš J. (2008): Influence of melatonin pretreatment and preconditioning by hypobaric hypoxia on the development of cortical photothrombotic ischemic lesion. Physiol. Res. 57, 283-288

Reiter R. J., Tan D. X., Leon J., Kilic U., Kilic E. (2005): When melatonin gets on your nerves: its beneficial actions in experimental models of stroke. Exp. Biol. Med. 230, 104-117

Reiter R. J., Tan D. X., Manchester L. C., Lopez-Burillo S., Sainz R. M., Mayo J. C. (2003): Melatonin: detoxification of oxygen and nitrogen-based toxic reactants. Adv. Exp. Med. Biol. 527, 539-548

Tan D. X., Manchester L. C., Sainz R. M., Mayo J. C., León J., Reiter R. J. (2005): Physiological ischemia/reperfusion phenomena and their relation to endogenous melatonin production: a hypothesis. Endocrine 27, 149-157; doi:10.1385/ENDO:27:2:149

Vannuci R. C., Vannuci S. J. (2005): Perinatal hypoxic-ischemic brain damage: evolution of an animal model. Dev. Neurosci. 27, 81-86; doi:10.1159/000085978

Received: April 4, 2009

Final version accepted: September 28, 2009 